\title{
Design of the Measurement and Control System for Deep-Sea Polymetallic Sulfide Sampler
}

\author{
Qiaodi Zhou ${ }^{1,2, a}$, Yi Tong ${ }^{1}$, Qingpeng Kong ${ }^{1}$, Haibin $\mathrm{Yu}^{1,2}$ \\ ${ }^{1}$ School of Electronic \& Information, Hangzhou Dianzi University, Hangzhou, 310018, China \\ ${ }^{2}$ School of Information Engineering, Hangzhou Dianzi University, Hangzhou, 310018, China \\ aemail: edizhou@163.com
}

Keywords: Measurement and Control; Deep-sea Sampling; Polymetallic Sulfide; STM32F103

\begin{abstract}
Large scale mining of deep-sea mineral resources is an important approach to solve the increasing scarcity of resources on Earth. The deep-sea polymetallic sulfide sampler is an effective device to realize the ore body mining of deep-sea polymetallic sulfide. In order to achieve stable remote communication and reliable control, this paper has developed a set of measurement and control system for this device. This system is mainly unitized by the above water monitoring platform, the underwater control center, the fiber-optic communication system and the matching monitoring software. The workshop test result shows that this system not only can monitor the important position of the underwater sampler but also can feed back the parametric data real-timely and accomplish the reliable control. At the same time, this system has good extensibility because of the CAN bus design and the abundant reserved interfaces.
\end{abstract}

\section{Introduction}

Earth's resources have caused international attention for several years. The developed countries are now moving their attention from the land to the ocean [1][2]. This makes the rapid development of marine science and technology. China started deep-sea sampling technique late. The marine scientific research and the marine resources exploration face severe difficulty. At present, the deep-sea sampling technique is developing in China, but we still have huge gap with the developed countries. The control system of the deep-sea polymetallic sulfide sampler which we discussed, from a technical and practical application level, belongs to the category of marine detection technology [3][4].

Measurement and control system of the deep-sea polymetallic sulfide sampler as a central control system of deep-sea equipment, the quality of its performance largely determines the outcome of sampling; it directly affects the actual mechanical motion of sampler, and the reliability of the whole system. At depth of 4000 meters in the ocean, the pressure is $40 \mathrm{Mpa}$, the temperature is often 1-2 degrees centigrade and there is almost no light [5]. Except these situations, the unpredictable strong oceanic currents which may do harm to the system are also a risk factor of the system. Because of these critical factors, the development of a low temperature resistance, high pressure resistance and shock resistance system has become an urgent desire.

In this paper the whole control scheme design of the deep-sea polymetallic sulfide sampler and its operation mechanism were described firstly. Then the design about the embedded system of the underwater control central was described. At the end the test results of this system was showed.

\section{Overview of the System}

The whole system consists of above-water monitoring platform subsystem with PC software, photoelectric composite cable communication subsystem, and the underwater control subsystem.

Above-water monitoring platform subsystem with PC software is on the ship in laboratory. Beside this computer we also have an optical transceiver which receive information from the optical cable and send instructions back to the optical cable. This part is the remote control terminal of the whole system. All mechanical actions can operate on the industrial computer which installs the 
customized software. Also we can monitor all important parameters. These parameters include AC voltage, AC current, GPS data from the ship, insulation value of the high voltage from the ground, altitude of the sampler from the sea-floor, temperature of the underwater measurement and control subsystem, depth of the sampler in the ocean, pressure values of the hydraulic system, images of the sampler scene, and so on.

The photoelectric composite cable [6] communication subsystem includes a winch and an armoured photoelectric composite cable. The winch can rise up and lower down the sampler through the cable. We use the armoured photoelectric composite cable to ensure the safe of the sampler and this cable can make it easily and simply to transmit the power and the information.

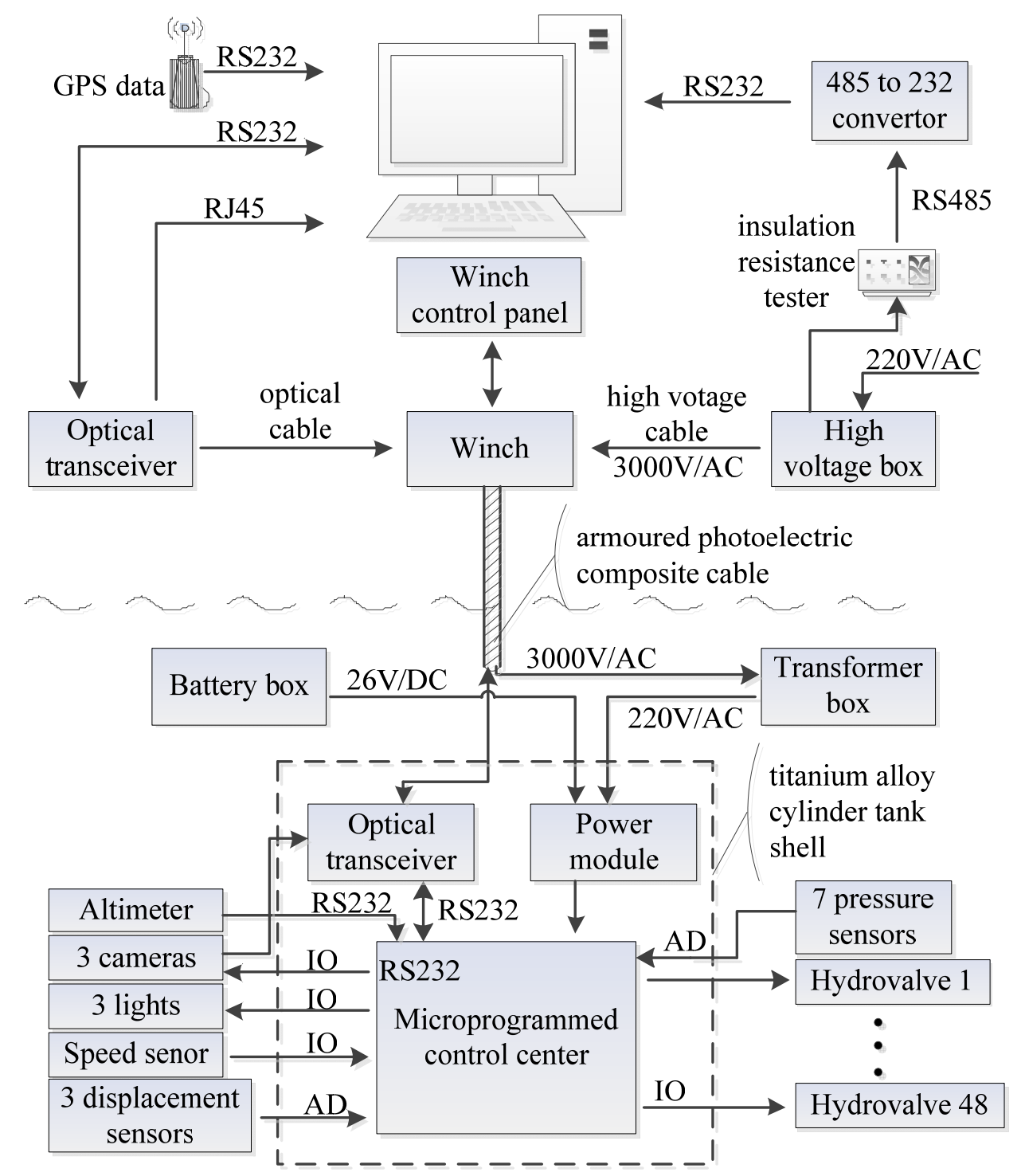

Fig.1. Overall scheme diagram

The underwater measurement and control subsystem is the core of the whole control system. All of the PCB we designed, the optical transceiver (corresponding with the above-water one) and the other modules are designed to put into a titanium alloy cylinder tank shell. We also have an underwater transformer box to decrease the alternating current from $3000 \mathrm{~V}$ to $220 \mathrm{~V}$ and an underwater battery box to start the system outside the tank shell. Almost every PCB uses DC power, so we commutate the $220 \mathrm{AC}$ to DC. Figure 1 shows the design of the whole scheme.

\section{Hardware Design}

\section{Power Module Design}

As we can see in the overall scheme, there are many hydraulic valves to be controlled. At the same time, we should turn off the lights, cameras and altimeter when the sampler is lowering down 
and turn on them when the sampler is almost closing to the sea-floor or working on the sea-floor. In order to ensure the power supply is adequate, we first need to calculate the maximum power consumption of the system. According to our design scheme, the power consumption for a single part and the quantity of each part are shown in table 1.

\begin{tabular}{|l|c|c|c|}
\hline & Power (W) & Quantity & Remark \\
\hline Light & 100 & 3 & \\
\hline Mother board & 140 & 1 & Including cameras, altitude and some sensors \\
\hline Sampling board & 15 & 1 & \\
\hline Extended board & 210 & 2 & 875 \\
\hline Total & \multicolumn{3}{|l}{} \\
\hline
\end{tabular}

Tab.1. Estimation of power consumption

Calculation result of the total power consumption is $875 \mathrm{~W}$. Because the space of the tank shell is narrow, so we should choose a kind of DC-DC module with high density. We choose the DC-DC module designed by the Vicor Corporation. One type is V300A24E600BG3, and another type is V24C24C100BL. Figure 2 shows the power module diagram. The vicor module has an important character of current sharing [7]. So we use two parallel $600 \mathrm{~W}$ modules to rise up the supply power to $1200 \mathrm{~W}$. It is sufficient for the whole system's power consumption. In consideration of the extended boards which are used to control the hydraulic valves and the valves' dynamic current is large, we use $26 \mathrm{~V} / \mathrm{DC}$ to decreasing the working current. At the same time, we use two $100 \mathrm{~W}$ modules to isolate the control circuit of the valves from the sampling circuit and analog circuit in order to eliminate unnecessary electromagnetic interference. The underwater battery box is mainly used to start up the system before we start the high-voltage power supply.

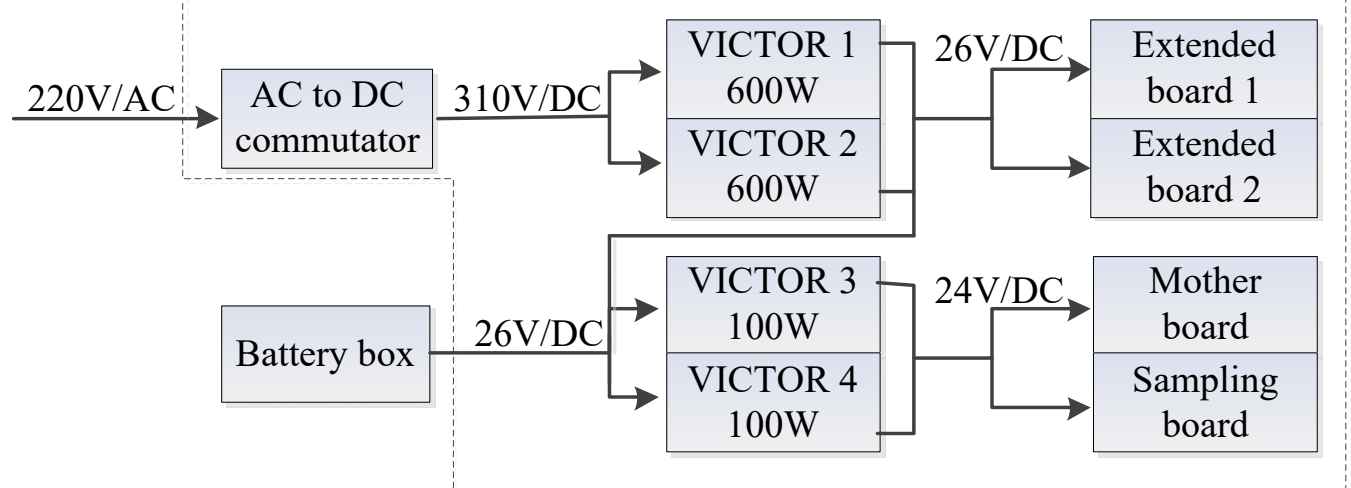

Fig.2. Power module diagram

\section{Measurement and Control Subsystem Circuit Design}

The measurement and control subsystem is the core of the whole system. In order to measure or monitor the states of the sampler, the subsystem should connect with multiple measuring sensors (altimeter, temperature, speed, pressure voltage, image, etc). In addition to measuring so many analog or digital values, we also should control amounts of hydraulic valves corresponding to different mechanical actions. In view of the above reasons, we adopt the divide-and-conquer method. We design 3 different PCB to accomplish different kind of tasks. Figure 3 shows the diagram of the measurement and control subsystem.

In this design, these three boards take STM32F103XX as the microprocessor, it is based on high-performance ARM Cortex-M3 32-bit RISC core operating at a frequency of up to $72 \mathrm{MHz}$. It has a built-in 512KB flash memory, a multi-channel ADC, 5 USART and several sets of I/O ports. Between these boards, we adopt CAN bus to communicate with each other. CAN bus is simple and reliable [8]. Basically, motherboard sends instructions to the other boards and receives information from other boards. At the end the useful information is packed and sent to the optical transceiver using one USART of the motherboard. The motherboard is designed to control cameras, lights and receive speed and altimeter data. All the analog values are collected to the sampling board, and all the hydraulic valves are controlled by the extended board. We will use two extended boards because one board's PCB layout could not satisfy 48 hydraulic valves' power consumption requirement. The 
DEBUG interface on these three boards is a RS232 interface. This reserved interface is used for test data, which makes the early test more intuitive and convenient.

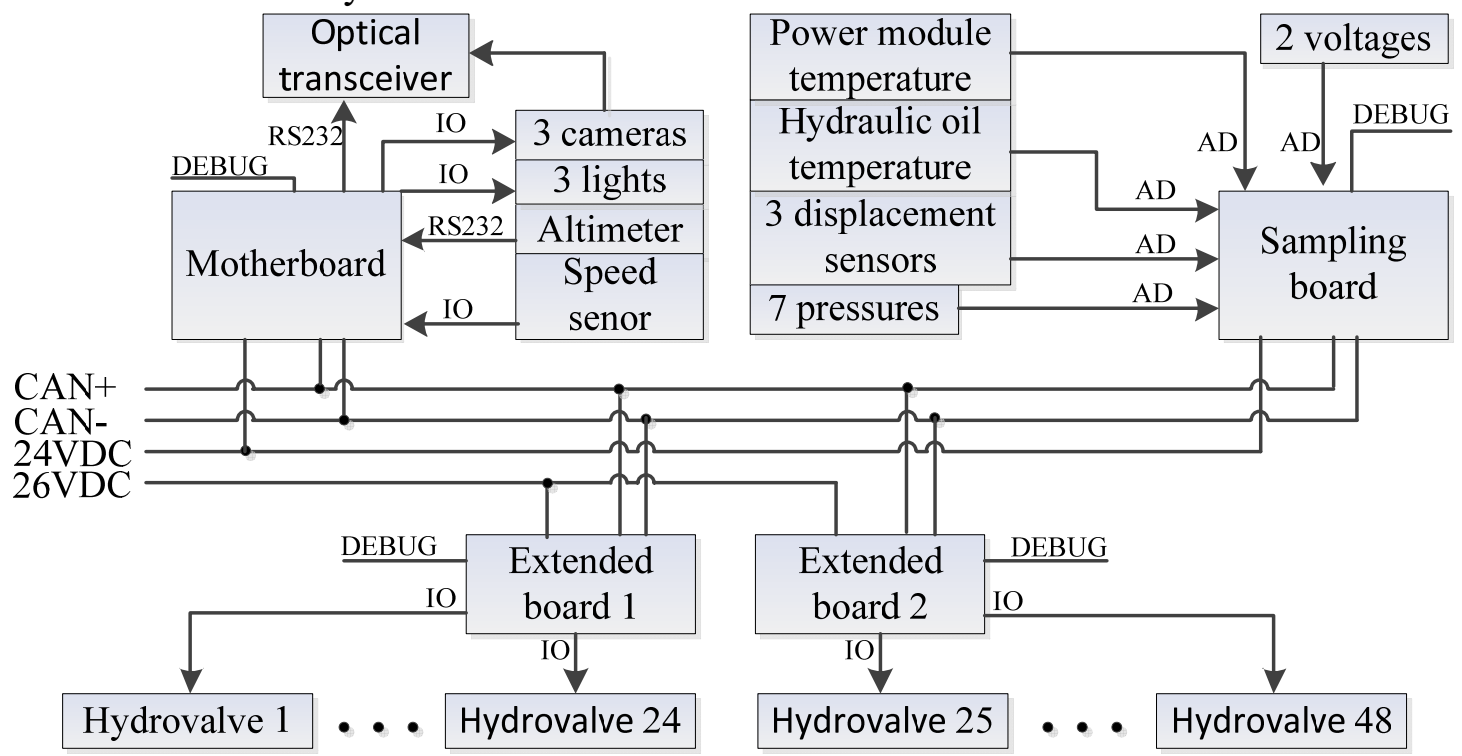

Fig.3. Measurement and control subsystem diagram

\section{Software Development}

Different board's embedded software does different jobs. The software installed on the PC is used to monitor all the parameters and control the actions of the sampler. Here we mainly discuss the embedded software of the underwater subsystem.

In the view of the CAN bus, the motherboard works as a master. The sampling board and the extended boards work as slaves. Within the perspective of USART, ADC or I/O, they are all master of themselves. The values that the sampling board get and the state of the relays on the extended board are sent to the motherboard through the CAN bus. The workflow of the subsystem's software can be described as follow:

1) Initialization and configuration for each board.

2) Extended boards wait for the commands from the motherboard. Sampling board will always sampling the analog values through the DMA channels. Motherboard will get the altimeter and the speed data.

3) When the motherboard gets a packaged information from the optical transceiver, it will analyse it. Then do some thing and send the analysed result to the real destination through the CAN bus. The board that gets the useful information will analyse it again and do some work or send the useful data back to the motherboard. The motherboard will pack these collected information and send them to the optical transceiver.

4) Cycle above two steps until the subsystem is powered off.

\section{Test results and Conclusion}

In the earliest testing stage, we just sent command to each of the boards through the DEBUG interface and used multimeter or the messages showing on the serial communication software to check the results. Then we connected all the boards together and ran the load test. We tested a full load of $900 \mathrm{~W}$ by using the high-power resistor. The load test results are shown in figure 4 . In figure 4, the T1 line is the temperature of the power module, the T2 line is the temperature of the MCU, and the T3 line is the environment temperature. The full load test shows that the system can work well under the condition of heavy load in room temperature, let alone in the ocean of 2-3 degrees centigrade. Finally, we went into the workshop and assembled our control system with the mechanical parts and the hydraulic parts. The final results show that this system has higher reliability and stability. And this system was checked and accepted by the China Ocean Mineral 
Resources R\&D Association. The overall constructed equipment is shown in Figure 5. Human-computer interaction interface which is the remote control center of this device is shown in figure 6 .

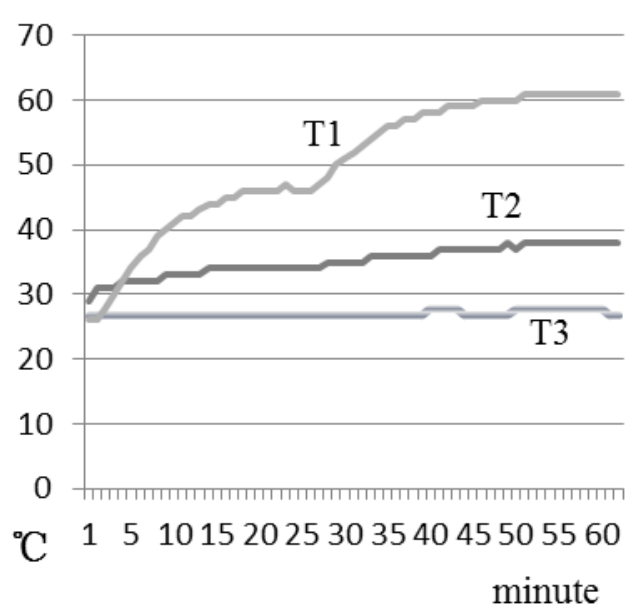

Fig.4. Load test result

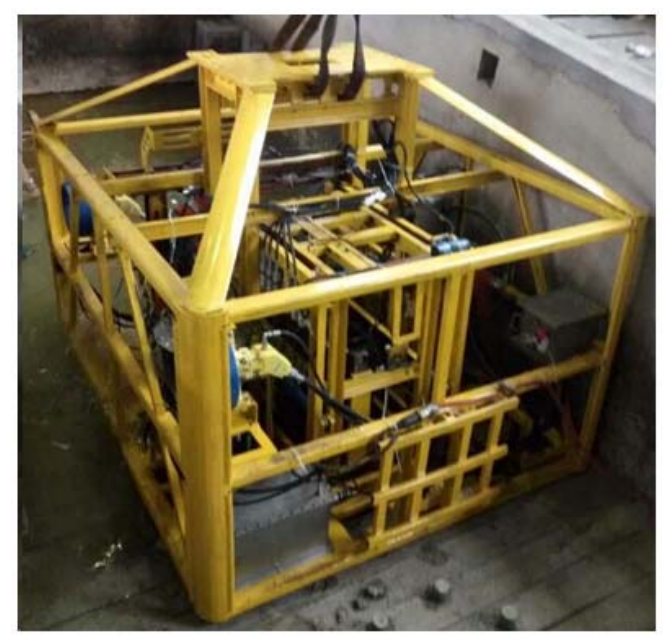

Fig.5. Whole underwater system

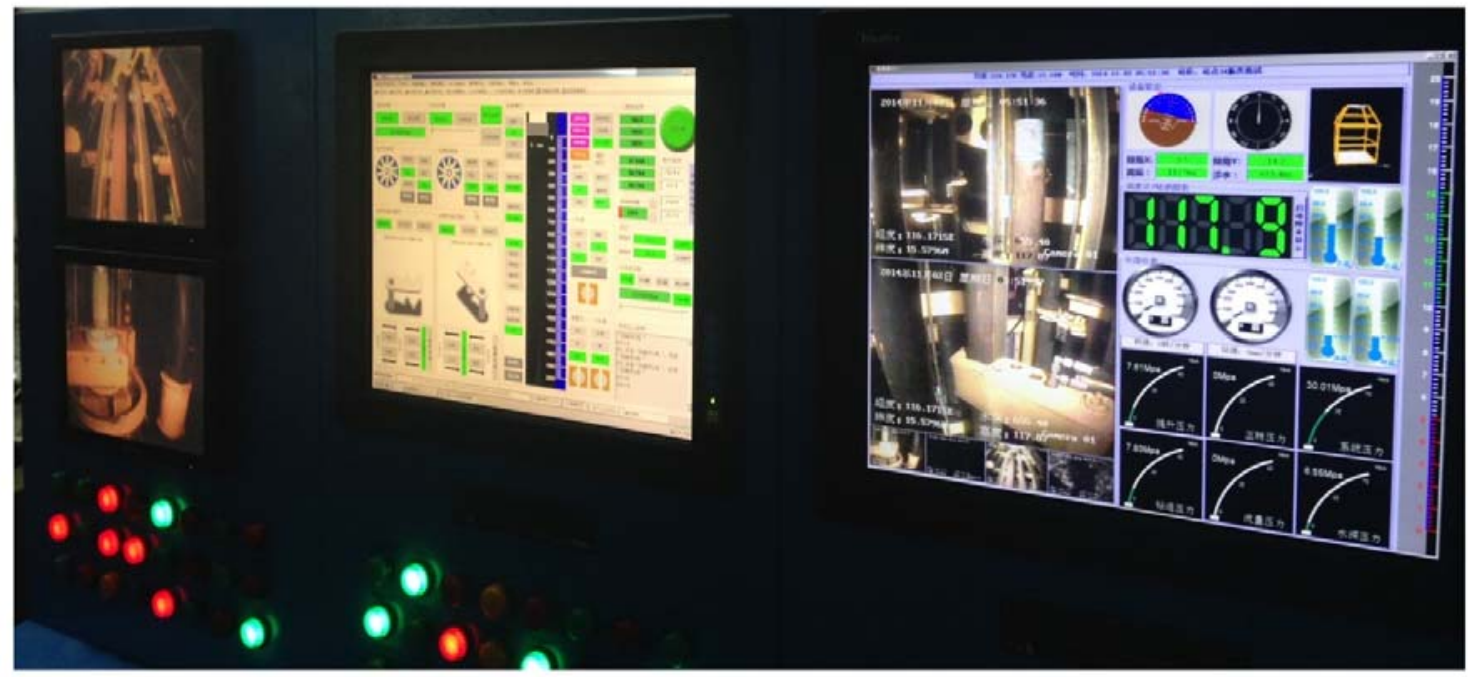

Fig.6. The remote control center

In conclusion, this system has multiple RS232 interfaces, multiple A/D interfaces available to sensors, and amounts of control value interfaces. The adequate and reliable power supply ensures that the whole measurement and control system can easily operate without too much burden. The CAN bus design makes every part of the system can simply connect and let the system has higher scalability and reliability.

\section{Acknowledgement}

In this paper, the work was partially supported by China Ocean Mineral Resources R\&D Association Funding Project (Grant No. DY125-11-T-01) and National High-tech R\&D Program of China (863 Program, Grant No. 2011AA090401). We gratefully thank the research team of Changsha Research Institute of Mining and Metallurgy which debugged the whole system with us in the workshop.

\section{References}

[1] Yuefeng Ni, Bo Lei, Guixiang Li. The Current Development Situation and Prospect of Marine Technology. [J]. Ocean Development and Management, 1999 (4) 40-44.

[2] Wenguang Zhu. The Development of Marine Hi-Technology in China. [J]. Ocean Technology, 
1998 (3) 7-12.

[3] Xiaomei Xu. Overview of Marine Technology. [M]. Science Press, 2000 10-45.

[4] Working Mechanism and selection principle of Seabed Sampler - The Second Subject of Seabed Sampling Technology. [J]. Exploration Engineering, 2001 (3) 19-22.

[5] Nybakken, James W. Marine Biology: An Ecological Approach. Fifth Edition. Benjamin Cummings, 2001 136-141.

[6] Jiping Xue, Chi Xue and so on. Submarine photoelectric composite cable non-universal optical unit. [P]. 2007.

[7] Vicor Application Engineer. Designing High-Power Arrays Using Maxi, Mini and Micro Family DC-DC Converters, 2013.

[8] Shangfeng Du, Xiaozhong Cao, Jin Xu. CAN Bus Control Technology and Application. [M]. Publishing House of Electronics Industry, 2007 9-13. 